\title{
RELEASE OF CARDIAC TROPONIN I IN ANTEGRADE CRYSTALLOID VERSUS COLD BLOOD CARDIOPLEGIA
}

M. Hendrikx, $\mathrm{MD}, \mathrm{PhD}^{\mathrm{a}, \mathrm{b}}$

H. Jiang, $\mathrm{MD}^{\mathrm{b}}$

H. Gutermann, $\mathrm{MD}^{\mathrm{b}}$

J. Toelsie, $\mathrm{MD}^{\mathrm{a}}$

D. Renard, $\mathrm{MSc}^{\mathrm{c}}$

A. Briers ${ }^{\mathrm{b}}$

J. L. Pauwels ${ }^{b}$

U. Mees, $\mathrm{MD}^{\mathrm{b}}$
Objective: The purpose of this study was to assess the efficacy of myocardial protection, comparing antegrade crystalloid cardioplegia with cold blood cardioplegia, in patients with preserved left ventricular function who were undergoing elective first coronary artery bypass grafting. Release of cardiac troponin I was used as a marker for the effectiveness of myocardial protection. Methods: A consecutive series of 62 patients were randomly assigned to receive crystalloid or blood cardioplegia. Cardiac troponin I concentrations were determined in venous blood samples before the operation, immediately after unclamping, at 6, 9, 12, and 24 hours, and daily thereafter for 5 days. Results: Rising levels of troponin I were found in all patients. The time course and peak release were similar in the crystalloid cardioplegia and the blood cardioplegia groups. No patients in either group had electrocardiographic evidence of perioperative myocardial infarction. Cardiac troponin I was able to detect small areas of myocardial damage, not revealed by electrocardiography or creatine kinase MB release. Aprotinin administration was associated with lower cardiac troponin I release in both groups. Cardiac troponin I was lower in patients whose conditions did not require electrical defibrillation after aortic unclamping, irrespective of cardioplegia type. The presence of a main stem lesion was associated with higher cardiac troponin I release only in the crystalloid cardioplegia group. Conclusions: Antegrade cold blood cardioplegia is equally effective as antegrade crystalloid cardioplegia in a randomized group of patients with preserved left ventricular function who were undergoing elective first coronary artery bypass grafting. Aprotinin administration resulted in lower cardiac troponin I release, whereas electrical defibrillation was related to a higher release irrespective of cardioplegia type. The presence of a main stem lesion resulted in higher cardiac troponin I release in the crystalloid cardioplegia group. (J Thorac Cardiovasc Surg 1999; 118:452-9)
Drev revious studies have frequently used postoperative electrocardiographic (ECG) changes and the release of cardiac enzymes, such as the MB isoenzyme of creatine kinase (CK-MB) for assessing the adequacy of myocardial protection during coronary artery bypass grafting $(\mathrm{CABG})$. The value of these markers in

From the Faculty of Medicine, ${ }^{\mathrm{a}}$ Limburgs Universitair Centrum, Diepenbeek, Belgium; Department of Cardiothoracic and Vascular

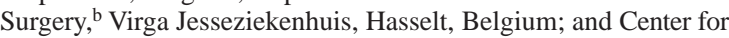
Statistics, ${ }^{c}$ Limburgs Universitair Centrum, Diepenbeek, Belgium.

Supported by a Research Fund of the Provincie Limburg ("Fonds Slimme Regio").

Received for publication Oct 26, 1998; revisions requested Jan 27, 1999; revisions received May 10, 1999; accepted for publication May 11, 1999. patients undergoing $\mathrm{CABG}$ is limited by the lack of sensitivity of ECG changes and by enzyme release from noncardiac tissue. ${ }^{1}$

The aim of this prospective, randomized study was to compare the use of antegrade crystalloid cardioplegia (CCP) with antegrade cold blood cardioplegia (BCP) in

\footnotetext{
Address for reprints: M. Hendrikx, MD, PhD, Department of Cardiothoracic and Vascular Surgery, Virga Jesseziekenhuis, Stadsomvaart, 11, B-3500 Hasselt, Belgium.
}

Copyright ㄷ 1999 by Mosby, Inc.

$0022-5223 / 99 \$ 8.00+0 \quad \mathbf{1 2 / 1 / 9 9 9 2 9}$ 
patients with preserved left ventricular function undergoing first-time elective CABG.

Cardiac troponin I (CTnI) was used as a biochemical marker for the adequacy of myocardial protection, because of its high sensitivity and specificity.

\section{Methods}

Patient selection. From January 5 through April 14, 1998, 115 CABG operations were performed at the Virga Jesse Hospital Hasselt by 2 staff surgeons (U.M. and M.H.). From these operations, 62 consecutive patients (49 men and 13 women; mean age, $67 \pm 7.7$ years) agreed to participate in a prospective, randomized trial comparing cold CCP with cold BCP.

Exclusion criteria for this study were patients with single vessel disease ( $\mathrm{n}=8$; all treated with minimally invasive direct coronary bypass [MIDCAB] procedures), patients with poor left ventricular function (left ventricular ejection fraction $<30 \% ; \mathrm{n}=32$ ), patients undergoing redo CABG surgery $(\mathrm{n}=11)$, and patients with concomitant heart valve disease. Two patients were excluded because of refusal to sign an informed consent form. Both cardioplegic techniques were used by both surgeons. The study was approved by the institutional human experimentation committee, and written consent was obtained from all patients.

Operative procedure. All operations were performed with cardiopulmonary bypass, with ascending aortic cannulation, 2stage venous cannulation, and moderate hypothermia $\left(32^{\circ} \mathrm{C}\right)$.

The left ventricle was vented by a catheter that was introduced through the right superior pulmonary vein.

In patients who had discontinued salicylic acid intake less than 10 days before the operation, aprotinin was administered according to the Hammersmith scheme. A loading dose of 2 million kallikrein inactivation units (KIU) was given before sternotomy, followed by an infusion of $0.5 \times 10^{6} \mathrm{KIU} / \mathrm{h}$ until the end of the operation. Additionally, 2 million KIU was added to the priming volume.

The route of cardioplegia delivery was exclusively antegrade in both groups.

Cardiac arrest was achieved by injecting an average of 750 $\mathrm{mL}$ of cardioplegic solution into the aortic root at $7^{\circ} \mathrm{C}$ immediately after aortic crossclamping, to obtain a myocardial septal temperature of $10^{\circ} \mathrm{C}$ to $12^{\circ} \mathrm{C}$.

The administration of the cardioplegic solution was pressure controlled in both groups. An aortic root catheter was inserted and attached to the cardioplegia line after deairing. Aortic root pressure was continuously monitored through a pressure line attached to a strain gauge. The cardioplegic solution (crystalloid or blood) was infused into the root at a pressure of 70 to $80 \mathrm{~mm} \mathrm{Hg}$. In the CCP group, flow rates of 250 to $300 \mathrm{~mL} / \mathrm{min}$ per square meter were obtained, whereas in the BCP group, flow rates were between 110 and 130 $\mathrm{mL} / \mathrm{min}$ per square meter, because of the difference in viscosity. In patients with severe ischemic disease, pressure was sometimes allowed to rise above $80 \mathrm{~mm} \mathrm{Hg}$, not to reduce the flow below $200 \mathrm{~mL} / \mathrm{min}$ per square meter in the CCP group
Table I. Cardioplegic solutions

\begin{tabular}{|c|c|c|c|}
\hline & $C C P$ & $\begin{array}{l}\text { BCP additive } \\
\text { composition }\end{array}$ & $\begin{array}{c}\text { Approximate } \\
\text { BCP final } \\
\text { concentration }\end{array}$ \\
\hline Sodium $(\mathrm{mmol} / \mathrm{L})$ & 139 & 116 & 139 \\
\hline Potassium (mmol/L) & 32 & 146 & 32 \\
\hline Magnesium (mmol/L) & 14 & 68 & 14 \\
\hline Calcium $(\mathrm{mmol} / \mathrm{L})$ & 2 & 2 & 2 \\
\hline Chloride (mmol/L) & 153 & 363 & 153 \\
\hline Bicarbonate $(\mathrm{mmol} / \mathrm{L})$ & 8 & 0 & 18 \\
\hline Hematocrit (\%) & 0 & 0 & 18 \\
\hline
\end{tabular}

${ }^{*}$ The BCP is a mixture (4:1 dilution) of the oxygenated blood of the patient and of a hyperkalemic crystalloid additive solution.

or $100 \mathrm{~mL} / \mathrm{min}$ per square meter in the BCP group. However, the fact that it took longer to administer BCP did not significantly affect the total duration of crossclamp time $(46 \pm 12$ minutes [standard deviation] in the CCP group vs $47 \pm 11$ minutes $[\mathrm{SD}]$ in the $\mathrm{BCP}$ group).

An additional dose of $300 \mathrm{~mL}$ was reinjected after each distal anastomosis, except the last one. Myocardial protection was completed by topical ice-cold water. Proximal anastomoses were completed on the beating heart with an aortic partial occlusion clamp.

Cardioplegia groups. The composition of the $\mathrm{CCP}$, the $\mathrm{BCP}$ additive composition, and the approximate final $\mathrm{BCP}$ composition are shown in Table I. BCP was made by adding oxygenated blood from the pump to a modified additive composition in a ratio of cardioplegic solution/blood of $1: 4$, with the Sarns conducer cardioplegia set 9457 (Sarns Inc/3M Health Care, Ann Arbor, Mich), to obtain a final potassium concentration similar to that of the crystalloid solution used. Cardioplegic solution $(750 \mathrm{~mL})$ was infused initially; the infusion $(300 \mathrm{~mL})$ was repeated after each distal anastomosis. No warm controlled reperfusion protocol was used in the BCP group, and the crossclamp was removed immediately after the last distal anastomosis.

Measurements of cardiac marker proteins. Serial venous blood samples were collected before the induction of anaesthesia, immediately after aortic unclamping, at $6,9,12$, and 24 hours after unclamping, and daily thereafter for 5 days.

CTnI concentrations were measured by a specific microparticle enzyme immunoassay developed by Abbott Diagnostics (Abbott Laboratories, Abbott Park, Ill).

Each standard (purified CTnI) or sample was incubated with microparticles coated with anti-TnI antibodies. An aliquot of the reaction mixture was transferred to a glass fiber matrix. Alkaline phosphatase-labeled anti-TnI antibodies were allowed to bind to the microparticle complex. The substrate 4-methylumbelleferyl phosphate was added, and the fluorescent product, methylumbelleferone, was measured by the assay. This assay, as opposed to most assays used previously, reflects the accurate quantification of both free and complexed TnI.

Reliable immunologic detection of $\mathrm{TnI}$ is possible only 
Table II. Patient profile

\begin{tabular}{llll}
\hline Variable & \multicolumn{1}{c}{ CCP* } & \multicolumn{1}{c}{ BCP } & P value \\
\hline Mean age (y) & $64.7(7.2)$ & $69.7(7.4)$ & .01 \\
Male & 28 & 21 & .2 \\
Ejection fraction & $65.6(13.6)$ & $67.8(13.4)$ & .46 \\
Anterior MI & 8 & 6 & .77 \\
Inferior MI & 11 & 12 & .61 \\
Diabetes & 4 & 6 & .5 \\
Hypertension & 14 & 13 & 1 \\
BSA & $1.88(0.14)$ & $1.83(0.18)$ & .13 \\
Crossclamp time (min) & $46(12)$ & $47(11)$ & .45 \\
ECC time (min) & $88(21)$ & $91(16)$ & .39 \\
LITA & 30 & 28 & 1 \\
LITA + RITA & 5 & 2 & .43 \\
VSM & 50 & 52 & .59 \\
Grafts/patient & $2.84 \pm 0.63$ & $2.76 \pm 0.64$ & .59
\end{tabular}

$M I$, Myocardial infarction; $B S A$, body surface area; $E C C$, extracorporeal circulation; LITA, left internal thoracic artery; RITA, right internal thoracic artery; $V S M$, vena saphena magna.

"Mean (SD) for continuous variables; frequency for discrete variables.

†Wilcoxon test for continuous and ordinal variables, Fisher's exact test for binary variables.

when antibodies recognize both free $\mathrm{TnI}$ and $\mathrm{TnI}$ complexed with other troponin components. It was shown that, in conditions of myocardial damage, TnI is released in the bloodstream not in free form but as a complex. Furthermore, from observation of patients having a myocardial infarction, it became clear that the ratio of total to free $\mathrm{CTnI}$ in the serum varies in the course of observation and is different in serum samples from different patients. ${ }^{2}$

CK-MB activities were measured after immunoinhibition at $25^{\circ} \mathrm{C}$ by means of an $\mathrm{N}$-acetylcysteine-activated, optimized ultraviolet test from Merck (Darmstadt, Germany). CK-MB was measured at 6 hours after operation and daily thereafter. CK-MB levels above $6 \%$ were interpreted as indicating perioperative myocardial infarction.

ECG. A 12-lead ECG was performed before the operation, on admission in the intensive care unit, and then daily after the operation for 5 days. All ECGs were read by one cardiologist, blinded to the study. Diagnostic criteria for perioperative myocardial infarction were new Q waves of $0.04 \mathrm{~mm}$ or more or a reduction in $\mathrm{R}$ waves of more than $25 \%$ in at least 2 leads.

Statistical analysis. For descriptive purposes (Table II), the Wilcoxon rank sum test (in the case of continuous and ordinal variables) and Fisher's exact test (in the case of categoric variables) were used when comparing the distribution of preoperative and operative data between the 2 groups.

To compare the time evolution between the 2 treatment groups, a model for longitudinal data was used. ${ }^{3}$ Specifically, a general linear model with first-order autoregressive correlation structure was used. A log-linear model for the variance was further used to account for variance heterogeneity over time. ${ }^{4}$ The data were log transformed to normalize their distribution, although this could not be fully achieved at later time occasions because of the high skewness of the data. This above model was used to test for the significance of the treatment effect over time and to investigate the influence of possible subject-specific factors, such as electrical defibrillation, aprotinin administration, and crossclamp time. The effect of these covariates on $\mathrm{CTnI}$ release was allowed to be different at each time occasion. All computations were performed by the SAS procedure MIXED (SAS Institute, Inc, Cary, NC). ${ }^{5}$

\section{Results}

Patient profile. One patient in the $\mathrm{BCP}$ group was excluded from analysis. The samples of the patient were not drawn. Consequently, the analysis included 29 patients in the BCP group. Preoperative and operative data in both CCP and BCP groups are shown in Table II. With the exception of age, the groups were well matched.

Preoperative ejection fraction was equivalent in both groups.

A preoperative anterior myocardial infarction was present in 8 patients in the CCP group versus 6 patients in the $\mathrm{BCP}$ group; a preoperative inferior infarction was present in 11 patients in the CCP group versus 12 patients in the BCP group.

In all but 1 patient in each group, at least one thoracic artery was used. In the CCP group, 1 patient did not receive a thoracic artery because of a critical left subclavian artery stenosis, detected on angiographic examination; in the BCP group 1 patient had only vein grafting because of a combination of diabetes, marked obesity, and advanced age. The number of saphenous veins and distal anastomoses was equivalent in both groups.

The average amount of CCP injected was $1025 \pm 308$ $\mathrm{mL}$ in the CCP group and $254 \pm 92 \mathrm{~mL}$ in the BCP group $(P=.026$; Wilcoxon $)$.

The intensive care unit stay was $28 \pm 11.9$ hours in the $\mathrm{CCP}$ group and $25 \pm 9.1$ hours in the BCP group $(P=.23)$.

Five patients in the CCP group and 7 patients in the BCP group did not require inotropic support. A low dose $(3-6 \mu \mathrm{g} / \mathrm{kg}$ per minute) of a combination of dopamine and dobutamine was used in 23 patients in the $\mathrm{CCP}$ group and in 18 patients in the $\mathrm{BCP}$ group. Higher doses were required in 4 patients in the CCP group $(7 \mu \mathrm{g} / \mathrm{kg}$ per minute total in 2 patients and 8 $\mu \mathrm{g} / \mathrm{kg}$ per minute in 2 patients) and 4 patients in the BCP group $(7 \mu \mathrm{g} / \mathrm{kg}$ per minute in 3 patients and 8 $\mu \mathrm{g} / \mathrm{kg}$ per minute in 1 patient).

Norepinephrine $(0.025-0.1 \mu \mathrm{g} / \mathrm{kg}$ per minute) was used as a vasoconstrictor in 4 patients in the $\mathrm{CCP}$ group and in 3 patients in the BCP group. There was no need for intra-aortic balloon pumping or mechanical support in any of the patients. 


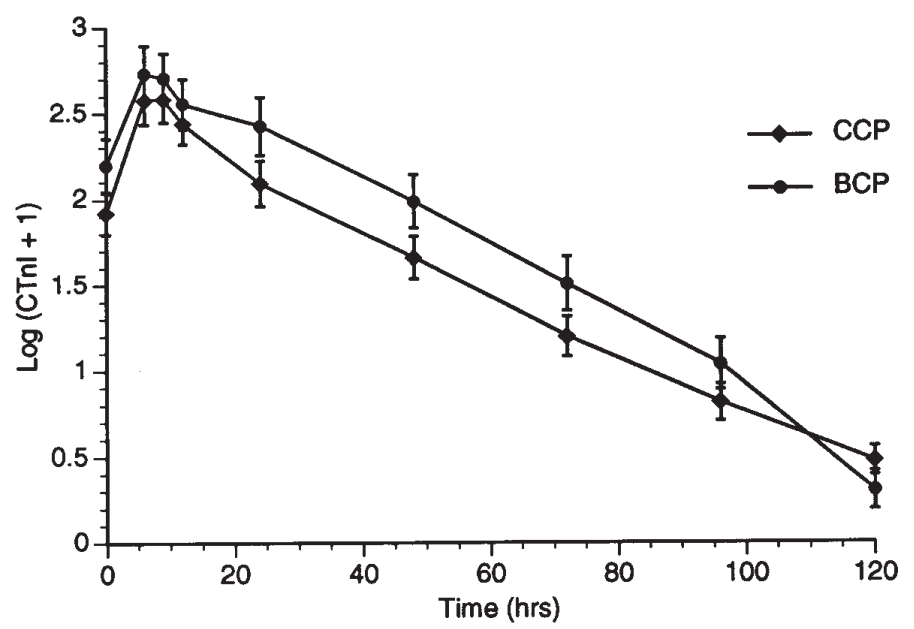

Fig 1. Time course of CTnI release according to the type of cardioplegia. $\log (\mathrm{CTnI}+1)$ was plotted as a function of time (hours). Time zero was chosen as the time of removal of the crossclamp. Error bars represent standard errors. The $P$ value for the comparison of these 2 profiles is .048 .

Peak CTnI release was not significantly different between patients requiring inotropic agents and patients who did not $(19.8 \pm 18.2 \mathrm{ng} / \mathrm{mL}$ vs $17.4 \pm 20.3$ $\mathrm{ng} / \mathrm{mL} ; P=.70)$. This indicates that inotropic agents were mainly used in the treatment of myocardial stunning rather than irreversible damage.

One patient in each group was in chronic atrial fibrillation before the operation. Acquired atrial fibrillation during the hospital stay occurred in 6 patients in the CCP group and in 8 patients in the BCP group. In the CCP group, 5 patients experienced the development of atrial fibrillation during their intensive care unit stay. Two patients were converted to sinus rhythm with amiodarone, and 2 patients were converted to sinus rhythm by propafenon. One patient needed electrical reconversion. One patient experienced the development of atrial fibrillation on the ward on postoperative day 4 . He was converted medically with propafenon.

In the BCP group, atrial fibrillation was treated successfully with amiodarone in 3 patients and by propafenon in another 3 patients. Electrical defibrillation was necessary in 2 patients. All patients were discharged in sinus rhythm. One patient in the CCP group experienced a transient ischemic attack, which fully recovered on postoperative day 4 . There were no cases of prolonged ventilatory support. There was no acquired left bundle branch block in either group. The angiographic distribution of coronary lesions did not differ between the 2 groups. There were no patients in either group with ECG evidence of perioperative myocardial infarction. The mean hospital stay was $8.7 \pm 1.2$ days in the CCP group and $8.6 \pm 0.9$ days in the BCP group $(P=.68)$.
Table III. $C K-M B$ values

\begin{tabular}{lcrc}
\hline & $C C P(S D)$ & $B C P(S D)$ & P value \\
\hline Before operation & $6.1(5.4)$ & $7.8(5.3)$ & .41 \\
After operation 6 hours & $22.6(13.1)$ & $22.3(8.5)$ & .85 \\
Postoperative day 2 & $14.8(6.4)$ & $15.8(9.0)$ & .66 \\
Postoperative day 3 & $12.9(5.6)$ & $15.7(7.7)$ & .12 \\
Postoperative day 4 & $12.1(14.3)$ & $11.7(6.6)$ & .9 \\
Postoperative day 5 & $10.8(15.0)$ & $9.1(6.6)$ & .64 \\
\hline
\end{tabular}

Comparison of CK-MB and CTnI release. This article reports on perioperative $\mathrm{CTnI}$ values in $\mathrm{CABG}$, with an assay that accurately measures both free and complexed CTnI. We used a cutoff value for CTnI (mean $\pm 2 \mathrm{SD}$ ) concentrations of $27.8 \mathrm{ng} / \mathrm{mL}$ immediately after aortic unclamping, $56.0 \mathrm{ng} / \mathrm{mL}$ at 6 hours after unclamping, $51.3 \mathrm{ng} / \mathrm{mL}$ at 9 hours after unclamping, and $40.1 \mathrm{ng} / \mathrm{mL}$ at 12 hours after unclamping.

According to those standards, 3 patients in the CCP and 4 patients in the BCP group had CTnI release above the cutoff values in at least 1 time point. We observed a prolonged increase in CTnI, rather than a distinct peak. In all those patients, CK-MB values were below the limit of $6 \%$ of total CK release.

$\mathrm{CK}-\mathrm{MB}$ values obtained in the $\mathrm{CCP}$ and $\mathrm{BCP}$ group are shown in Table III. At no point in time was there a significant difference between the 2 treatment groups.

BCP versus CCP. Fig 1 shows the time course of CTnI concentration for both groups of cardioplegia. CTnI concentration increased significantly $(P<.0001)$ from preoperative values starting at aortic unclamping in both groups. After an initial rise attaining its peak at 


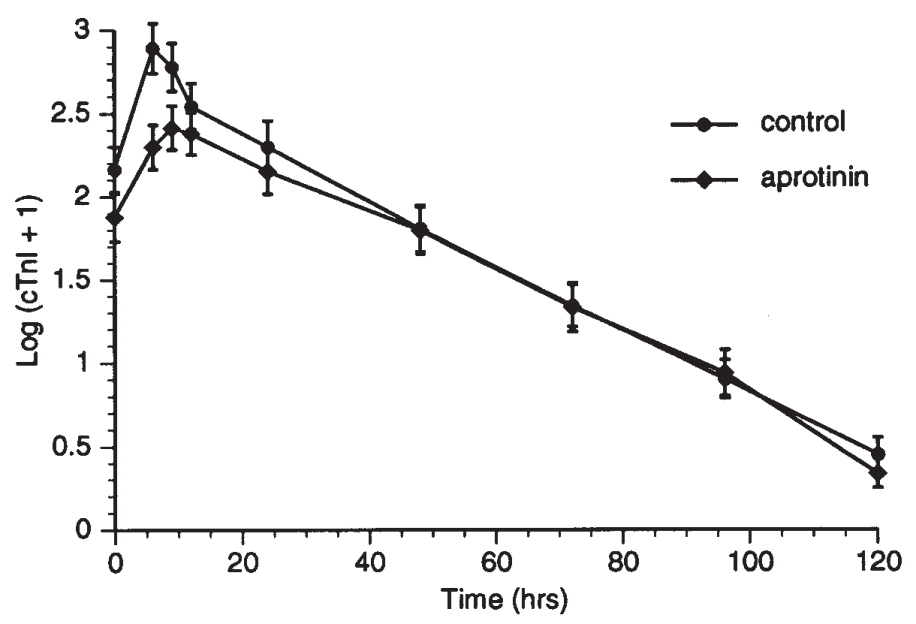

Fig 2. Time course of CTnI release according to whether aprotinin was administered (aprotinin) or not (control). $\log (\mathrm{CTnI}+1)$ was plotted as a function of time (hours). Time zero was chosen as the time of removal of the crossclamp. Error bars represent standard errors. Total CTnI release was significantly lower in the aprotinin group $(P=.0003)$. CTnI concentration at hours 6 and 9 was significantly lower in the aprotinin group.

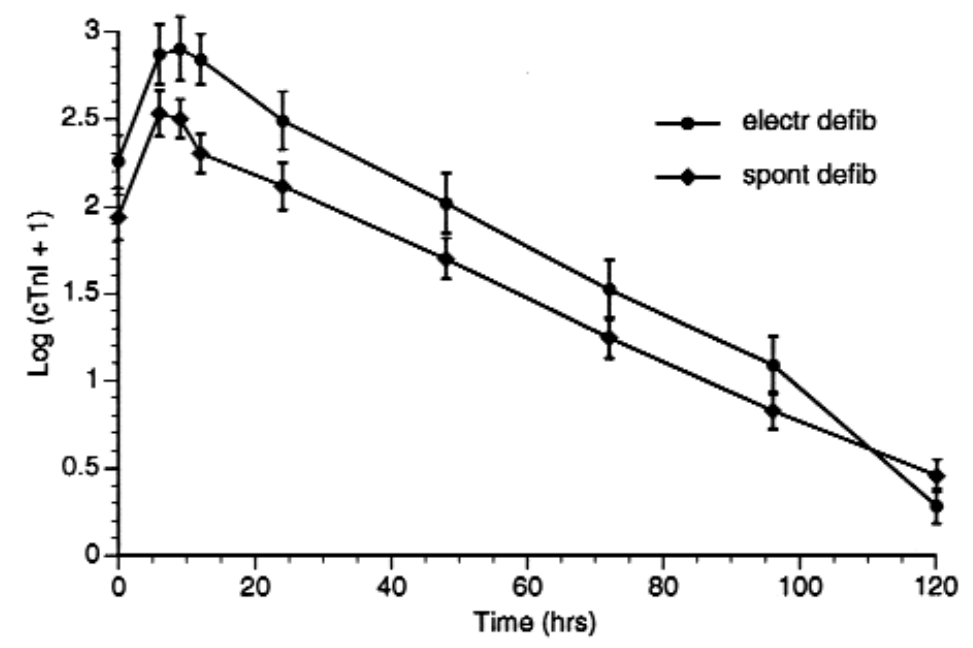

Fig 3. Time course of CTnI release according to cardiac rhythm recovery. Spont defib, Spontaneous defibrillation; electr defib, patients requiring electrical defibrillation. $\log (\mathrm{CTnI}+1)$ was plotted as a function of time (hours). Time zero was chosen as the time of removal of the crossclamp. Error bars represent standard errors. Overall CTnI release was significantly higher in the electrical defibrillation group $(P=.0057)$. CTnI concentration at 12 hours was significantly higher in patients needing electrical defibrillation.

6 hours after the operation, the level of CTnI constantly decreased over time. When the overall curves were considered, there was no clear difference between the 2 profiles $(P=.048)$. When a constant treatment effect over time was considered, the difference between the 2 treatment groups was not significant (unadjusted, $P=$ .31 ; adjusted, $P=.59$ ). The adjustment was for the need of electrical defibrillation, aprotinin administration, and crossclamp time, with an effect allowed to be different at each time occasion.
Effect of aprotinin. Because of recent salicylic acid intake, aprotinin was administered to 16 patients in the CCP group and 12 patients in the BCP group. Total CTnI release was significantly lower in the group of patients receiving aprotinin during the intervention $(P=.0003)$. CTnI levels were significantly lower in the aprotinin group at 6 hours $(11.7 \pm 9.2$ $\mathrm{ng} / \mathrm{mL}$ vs $25.3 \pm 21.8 \mathrm{ng} / \mathrm{mL} ; P=.002$, Wilcoxon) and at 9 hours $(13.1 \pm 10.3 \mathrm{ng} / \mathrm{mL}$ vs $22.1 \pm 19.8$ $\mathrm{ng} / \mathrm{mL} ; P=.041 ;$ Fig 2$)$. 


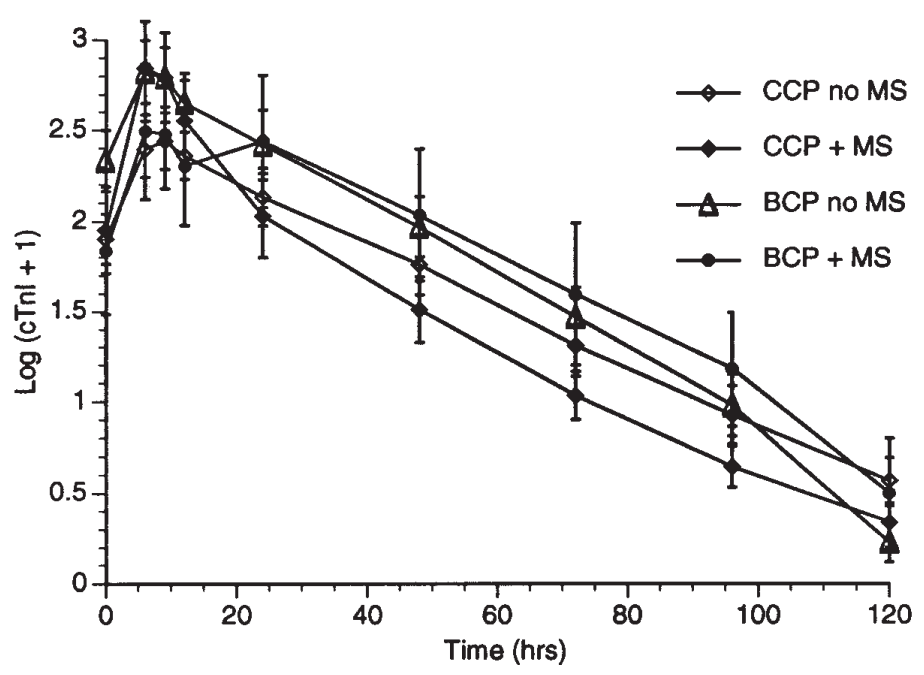

Fig 4. Time course of CTnI release according to the type of cardioplegia and whether a main stem lesion was present $(+M S)$ or absent $($ no $M S)$. $\log (\mathrm{CTnI}+1)$ was plotted as a function of time (hours). Time zero was chosen as the time of removal of the crossclamp. Error bars represent standard errors. In the CCP group, the overall curve of $\mathrm{CTnI}$ release was significantly higher in patients with a main stem lesion $(P=.0473)$. In the BCP group, no difference was observed between patients with or without main stem lesion $(P=.7060)$.

Electrical defibrillation and CTnI release. Electrical defibrillation was required in 10 patients in the $\mathrm{CCP}$ group and in 12 patients in the $\mathrm{BCP}$ group after removal of the aortic crossclamp to allow spontaneous electrical activity to resume. Overall, CTnI release was significantly higher in the group of patients who needed electrical defibrillation $(P=.0057)$. CTnI concentration was significantly higher in the defibrillation group at 12 hours $(11.7 \pm 9.8 \mathrm{ng} / \mathrm{mL}$ vs $20.3 \pm 15.2$ $\mathrm{ng} / \mathrm{mL} ; P=.005$, Wilcoxon; Fig 3).

Main stem lesions. A main stem lesion was present in 13 patients in the CCP group and in 8 patients in the $\mathrm{BCP}$ group. When the overall curve is considered, $\mathrm{CTnI}$ release was marginally significantly higher in patients with a main stem lesion in the CCP group when compared with patients who were treated for more distal lesions with CCP $(P=.0473)$. This difference was not present in the BCP group $(P=.4878$; Fig 4$)$.

Crossclamp time. In the multivariate analysis, the effect of aortic crossclamp time on CTnI release was significant at 24 hours $(P=.015)$, at 48 hours $(P=$ $.003)$, at 72 hours $(P=.006)$, and at 96 hours $(P=.009)$.

\section{Discussion}

The use of BCP has gained widespread acceptance. In a recent national survey, Robinson and colleagues ${ }^{6}$ reported that $\mathrm{BCP}$ was used by $72 \%$ of all respondent surgeons. On the other hand, the numerous methods of myocardial protection used indicate that there is no clear evidence of superiority of one method over another. Previous clinical studies, comparing BCP and CCP, have assessed the extent of myocardial damage by analyzing postoperative ECG changes or release of CKMB. However, the sensitivity of ECG changes in patients undergoing CABG is low. ${ }^{7}$ Therefore no conclusions can be drawn from the fact that there were no perioperative myocardial infarctions in either group according to ECG criteria. Also, no difference in CKMB release was found between the 2 groups at any point in time.

This study confirms previous reports, which show a substantial release of CTnI in elective CABG operations. ${ }^{8,9}$ The peak and total $\mathrm{CTnI}$ release was not significantly different in the BCP and CCP groups. This suggests that the damage caused to the myocardium in an unselected group of patients who were undergoing low-risk primary CABG is similar with both methods of cardioplegia. Our data confirm 1 previous report by Caputo and colleagues ${ }^{10}$ who also found no difference in $\mathrm{CTnI}$ release when comparing a group of patients receiving $\mathrm{BCP}$ with a group of patients receiving $\mathrm{CCP}$ with the use of an operative procedure similar to the one reported in this article.

However, absolute values of CTnI release are markedly higher than in their report. Recently, Katrukha and colleagues ${ }^{2}$ have reported that the largest part of $\mathrm{CTnI}$ is liberated as a complex, probably with troponin $\mathrm{C}$, and only a small part of $\mathrm{CTnI}$ circulates in 
a free form. It should be noted that the ratio of total to free CTnI varies between patients and within the course of each episode of myocardial damage. Therefore we selected a test that allows accurate quantification of both free and complexed troponin I, because it is unclear from the data by Caputo and colleagues ${ }^{10}$ whether the total CTnI release was similar in both groups or only the part liberated as free form.

Pichon and colleagues, ${ }^{11}$ on the other hand, have reported that the total amount of $\mathrm{CTnI}$ released was significantly higher in a CCP group than in a $\mathrm{BCP}$ group. However, their groups differed not only with respect to the type of cardioplegia but also by the fact that warm reperfusion was performed only in the BCP group. This warm controlled reperfusion may be beneficial, not only by better preservation of high-energy phosphates ${ }^{12}$ but also by limiting the transsarcolemmal $\mathrm{pH}$ gradient on reperfusion, hence limiting $\mathrm{Na}^{+}$and subsequent $\mathrm{Ca}^{2+}$ overload during reperfusion. ${ }^{13}$

The mechanisms underlying the protective effect of aprotinin on the myocardium remain unclear. To rule out an effect of aprotinin on the CTnI assay itself, aprotinin $(300 \mathrm{KIU} / \mathrm{mL})$ was added to the plasma of patients with known CTnI elevation in the same range as the one seen after cardiopulmonary bypass. The concentration of aprotinin was chosen to be at the upper range of measured aprotinin concentrations at the initiation of cardiopulmonary bypass. ${ }^{14}$ After adding aprotinin to the plasma, measured CTnI levels did not change beyond the dilution factor. Also Wendel and colleagues ${ }^{15}$ reported lower cardiac troponin $\mathrm{T}$ levels in patients undergoing cardiopulmonary bypass receiving high-dose aprotinin therapy. Cardiac operations with cardiopulmonary bypass are associated with the release of various inflammatory mediators such as tumor necrosis factor $\alpha$. Integrin CD11b is a neutrophil surface adhesive glycoprotein that becomes rapidly and irreversibly unregulated by tumor necrosis factor $\alpha$. Hill and colleagues ${ }^{16}$ have reported that even low-dose aprotinin has a significant effect in blunting systemic tumor necrosis factor $\alpha$ release and neutrophil integrin CD11b up-regulation. Furthermore, full-dose aprotinin has been reported to reduce the cardiopulmonaryinduced release of the proinflammatory cytokine, interleukin- $6,{ }^{17}$ and enhance the release of the endogenous anti-inflammatory cytokine, interleukin-10. ${ }^{18}$

However, this cannot be the only mechanism because the protective action of aprotinin was shown both in the isolated rat heart subjected to global ischemia ${ }^{19}$ and in a model of regional ischemia in the dog. ${ }^{20}$ Oxygen free radicals, derived in part from neutrophils and formed particularly during the reperfusion phase, may con- tribute to membrane damage by activation of proteases. Treatment with aprotinin would adequately prevent a hydrogen peroxide-induced fall in catalase activity and hence reduce the magnitude of cytotoxicity. ${ }^{21}$ This would ultimately result in better preservation of selective sarcolemmal permeability and reduced cellular calcium overload.

Recently, the results of the first international multicenter trial, designed to address the graft occlusion problem associated with aprotinin administration, with graft angiography (IMAGE trial), have been reported. ${ }^{22}$ The probability of early vein graft occlusion was increased by aprotinin $(15.4 \%$ of the patients receiving aprotinin versus $10.9 \%$ of patients receiving placebo; $P$ $=.03$ ), whereas thoracic artery patency was not affected. Subanalysis revealed several risk factors for early graft failure: aprotinin definitely promotes graft occlusion in women (5-fold increase over placebo), in smaller vessels $(<1.5 \mathrm{~mm})$, and in patients with poor-quality distal vessels. Therefore the routine use in coronary surgical procedures should not be advocated. However, careful selection of patients based on the known risk factors of early graft failure associated with aprotinin use would allow a subgroup of patients to benefit from the protective action of aprotinin during ischemic arrest.

$\mathrm{CTnI}$ concentration was significantly lower at 12 hours after unclamping in patients not requiring electrical defibrillation, which is consistent with a previous report by Pichon and colleagues. ${ }^{11}$ However, the striking difference is that, in their CCP group, all but 1 patient of 35 required electrical defibrillation, whereas in the BCP group 17 patients did not. In this study, the need for electrical defibrillation was evenly distributed between the $\mathrm{BCP}$ and the CCP group: 12 patients in the $\mathrm{BCP}$ group and 10 patients in the $\mathrm{CCP}$ group required electrical defibrillation. This suggests that the reduced need for electrical defibrillation observed by Pichon and colleagues ${ }^{11}$ is a consequence of warm reperfusion, rather than the type of cardioplegic solution used.

$\mathrm{CTnI}$ release was significantly higher in the CCP group in patients with a critical main stem lesion. In the BCP group, CTnI release was similar whether a main stem lesion was present or not. It would indicate improved protection with $\mathrm{BCP}$ in the subgroup of main stem patients. This may be explained by the fact that perfusion, distal to coronary stenoses, was shown to be improved with blood as opposed to CCP. ${ }^{23}$

In this study, the effect of aortic crossclamp time was significant at a few time points. This effect, however, was not as strong as could be expected. This may be explained by the fact that myocardial damage in patients undergoing CABG is multifactorial. The 
anatomy of the coronary lesions may variably influence the efficacy of antegrade cardioplegia.

\section{Conclusion}

Rising levels of CTnI are found in all patients undergoing $\mathrm{CABG}$, with peak concentrations at 6 hours after aortic unclamping. This specific and sensitive marker does not detect significant difference in myocardial protection between antegrade cold CCP and antegrade cold $\mathrm{BCP}$ in patients with preserved left ventricular function undergoing elective first-time CABG. High-dose aprotinin administration was associated with lower CTnI release, suggesting protective action against ischemic injury. Electrical defibrillation resulted in higher CTnI release in both $\mathrm{CCP}$ and BCP groups. Higher CTnI levels were observed in patients with a main stem lesion only in the CCP group. This would indicate improved protection with $\mathrm{BCP}$ in this subgroup of patients.

We thank Drs J. Kortleven and V. Peeters, for their help with troponin I measurements, and the team of cardiac anesthesiologists and nursing staff on the intensive care unit for their meticulous sampling of the data.

\section{REFERENCES}

1. Baldermann SC, Bhayana JN, Steinbach JJ. Perioperative myocardial infarction: a diagnostic dilemma. Ann Thorac Surg 1980;30:370-7.

2. Katrukha AG, Bereznikova AV, Esakova TV, Pettersson K, Lövgren T, Severina ME, et al. Troponin I is released in bloodstream of patients with acute myocardial infarction not in free form but as complex. Clin Chem 1997;43:1379-85.

3. Diggle PJ, Liang K-Y, Zeger SL. Analysis of longitudinal data. Oxford: Oxford Science Publications; 1994.

4. Littell RC, Milliken GA, Stroup WW, Wolfinger RD. SAS system for mixed models. Cary (NC): SAS Institute Inc; 1996. p. 294-301.

5. SAS/STAT software. Changes and enhancements through release 6.11. Cary (NC): SAS Institute Inc; 1996. p. 533-656.

6. Robinson LA, Schwarz GD, Doddard DB, Fleming WH, Galbraith TA. Myocardial protection for acquired heart disease surgery: results of a national survey. Ann Thorac Surg 1995;59: 361-72.

7. McGregor CGA, Muir AL, Smith AF. Myocardial infarction related to coronary artery bypass graft. Br Heart J 1984;51:399-406.

8. Etievent J-P, Chocron S, Toubin G, Taberlet C, Alwan K, Clement $\mathrm{F}$, et al. Use of cardiac troponin I as a marker of perioperative myocardial ischemia. Ann Thorac Surg 1995;59:1192-4.

9. Mair J, Larue C, Mair P, Balogh D, Calzori C, Puschendorf B. Use of cardiac troponin I to diagnose perioperative myocardial infarction in coronary artery bypass graft. Clin Chem 1994;40: 2066-70.
10. Caputo M, Dihmis W, Birdi I, Reeves B, Suleiman M-S, Angelini $\mathrm{GD}$, et al. Cardiac troponin $\mathrm{T}$ and troponin I release during coronary artery surgery using cold crystalloid and cold blood cardioplegia. Eur J Cardiothorac Surg 1997;12:254-60.

11. Pichon H, Chocron S, Alwan K, Toubin G, Kaili D, Falcoz P, et al. Crystalloid versus cold blood cardioplegia and cardiac troponin I release. Circulation 1997;96:316-20.

12. Teoh KH, Christakis GT, Weisel RD, Fremes SE, Mickle DAG, Romashin AD, et al. Accelerated myocardial metabolic recovery with terminal warm blood cardioplegia. J Thorac Cardiovasc Surg 1986;91:888-95.

13. Hendrikx M, Mubagwa K, Verdonck F, Overloop K, Van Hecke P, Vanstapel F, et al. New $\mathrm{Na}^{+}-\mathrm{H}^{+}$exchange inhibitor HOE 694 improves postischemic function and high-energy phosphate resynthesis and reduces $\mathrm{Ca}^{2+}$ overload in isolated perfused rabbit hearts. Circulation 1994;89:2787-98.

14. Bennett-Guerrero E, Sorohan JG, Howell ST, Ayuso L, Cardigan RA, Newman MF, et al. Maintenance of therapeutic plasma aprotinin levels during prolonged cardiopulmonary bypass using a large-dose regimen. Anesth Analg 1996;83:1189-92.

15. Wendel HP, Heller W, Michel J, Mayer G, Ochsenfahrt C, Graeter $\mathrm{U}$, et al. Lower cardiac troponin $\mathrm{T}$ levels in patients undergoing cardiopulmonary bypass and receiving high-dose aprotinin therapy indicate reduction of perioperative myocardial damage. J Thorac Cardiovasc Surg 1995;109:1164-72.

16. Hill GE, Alonso A, Spurzem JR. Stammers AH, Robbins RA. Aprotinin and methylprednisolone equally blunt cardiopulmonary-induced inflammation in humans. J Thorac Cardiovasc Surg 1995;110:1658-62.

17. Diego RP, Mihalakakos PJ, Hexum TD, Hill GE. Methylprednisolone and full-dose aprotinin reduce reperfusion injury after cardiopulmonary bypass. J Cardiothorac Vasc Anesth 1997; 11:29-31.

18. Hill GE, Diego RP, Stammers AH, Huffman SM, Pohorecki R. Aprotinin enhances the endogenous release of interleukin-10 after cardiac operations. Ann Thorac Surg 1998;65:66-9.

19. Gurevitch J, Barak J, Hochhauser E, Paz Y, Yakirevich V. Aprotinin improves myocardial recovery after ischemia and reperfusion.: effects of the drug on isolated rat hearts. J Thorac Cardiovasc Surg 1994;108:109-18.

20. Diaz PE, Fishbein MC, Davis HA, Askenazi J, Maroko PR. Effect of the kallikrein inhibitor aprotinin on myocardial ischemic injury after coronary occlusion in the dog. Am J Cardiol 1977;40:541-9.

21. Horwitz LD, Leff JA. Catalase and hydrogen peroxide cytotoxicity in cultured cardiac myocytes. J Mol Cell Cardiol 1995;27: 909-15.

22. Alderman EL, Levy JH, Rich JB, Nili M, Vidne B, Schaff H, et al. Analyses of coronary graft patency after aprotinin use: results from the international multicenter aprotinin graft patency experience (IMAGE) trial. J Thorac Cardiovasc Surg 1998;116:71630.

23. Robertson JM, Buckberg GB, Vinten-Johansen J, Leaf JD. Comparison of distribution beyond coronary stenoses of blood and asanguinous cardioplegic solution. J Thorac Cardiovasc Surg $1983 ; 86: 80-8$. 